\title{
Unionsbürgerschaft an der Schnittstelle zwischen Integration, Solidarität und sozialem Zusammenhalt
}

\author{
Dr Kathrin Hamenstädt
}

Der Gerichtshof der Europäischen Union hat in den letzten Jahren eine

konzeptionelle Kehrtwende in seiner Rechtsprechung zur Unionsbürgerschaft vollzogen. Der nachfolgende Beitrag soll der Frage nachgehen, welche Rolle die Konzepte der Integration, des sozialen Zusammenhalts und der Solidarität bei diesem Rechtsprechungswechsel spielen und wie die Interpretation und die Verwendung dieser Konzepte durch den Gerichtshof den Gehalt der Unionsbürgerschaft umgestalten. Dabei richtet sich der Fokus auf das Ausweisungsrecht, das Daueraufenthaltsrecht und den Zugang $\mathrm{zu}$ beitragsunabhängigen Sozialleistungen.

\section{Einleitung}

Die Staatsangehörigen der 28 Mitgliedstaaten der Europäischen Union besitzen neben ihrer jeweiligen Staatsangehörigkeit auch die durch den Vertrag von Maastricht eingeführte Unionsbürgerschaft. ${ }^{1}$ Die Unionsbürgerschaft erlaubt es ihnen sich unabhängig von einer wirtschaftlichen Tätigkeit frei im Gebiet der Europäischen Union zu bewegen und aufzuhalten ${ }^{2}$, wobei es einen Vorbehalt hinsichtlich ,der in den Verträgen und in den Durchführungsvorschriften vorgesehenen Beschränkungen und Bedingungen“" gibt. ${ }^{3}$ Neben der Unionsbürgerschaft

1 Art. 20 Abs. 1 Konsolidierte Fassung des Vertrags über die Arbeitsweise der Europäischen Union, AB1. 2012, C 326/01 (AEUV).

${ }^{2}$ Siehe Art. 20 Abs. 2 lit. a, Art. 21 Abs. 1 AEUV (Fn. 1), eine Garantie, die ebenfalls von Art. 45 Abs. 1 Charter der Grundrechte der Europäischen Union, ABl. 2012, C 326/326 (Grundrechtecharta) unterstrichen wird.

${ }^{3}$ Siehe Art. 21 Abs. 1 AEUV (Fn. 1). Für die Unterscheidung zwischen Bedingung und Beschränkung siehe $M$. Wendel, § 18 Unionsbürgerrechte, Freizügigkeit, in: 
ist im zweiten Teil des AEUV $^{4}$ der Grundsatz der Nichtdiskriminierung geregelt, welcher vorsieht, dass „(u)nbeschadet besonderer Bestimmungen der Verträge (...) in ihrem Anwendungsbereich jede Diskriminierung aus Gründen der Staatsangehörigkeit verboten" ist. ${ }^{5}$

Die Rechtsprechung des Gerichtshofs der Europäischen Union (EuGH) füllte das Konzept der Unionsbürgerschaft, oft unter Rückgriff auf den Grundsatz der Gleichbehandlung von Unionsbürger*innen mit Staatsangehörigen des jeweiligen Mitgliedstaates, mit Leben und trug maßgeblich zu dessen Entwicklung bei. ${ }^{6}$ In seiner vielzitierten GrzelczykEntscheidung urteilte der EuGH, dass der Unionsbürgerstatus dazu bestimmt sei „der grundlegende Status der Angehörigen der Mitgliedstaaten zu sein, der es denjenigen unter ihnen, die sich in der gleichen Situation befinden, erlaubt, unabhängig von ihrer Staatsangehörigkeit und unbeschadet der insoweit ausdrücklich vorgesehenen Ausnahmen die gleiche rechtliche Behandlung zu genießen“.7 Die Grzelczyk-Entscheidung fügt sich in eine Reihe weiterer Entscheidungen, wie Martínez Sala ${ }^{8}$, $D^{\prime} H_{o o p}{ }^{9}, M R A X^{10}$ und Baumbast und $R,{ }^{11}$ ein, in denen der Gerichtshof nicht nur die Rechte der Unionsbürger*innen, sondern auch ihrer (drittstaatsangehörigen) Familienmitglieder gefestigt und gestärkt hat, und die von Spaventa als konstituierende Phase ${ }^{12}$ bezeichnet wird. Huber betont, dass diese Entscheidungen für eine „Inklusion der von ihrem Freizügigkeitsrecht Gebrauch machenden Unionsbürger und (...) eine

Grabenwarter (Hrsg.): Enzyklopädie Europarecht, Bd. 2: Europäischer Grundrechteschutz (2014), S. 760.

${ }^{4}$ Vertrag über die Arbeitsweise der Europäischen Union (Fn. 1).

${ }^{5}$ Art. 18 Abs. 1 AEUV (Fn. 1).

${ }^{6}$ S. Giubboni, Free Movement of Persons and European Solidarity, ELJ 2007, 360, 369; Th. Kingreen, In love with the single market? Die EuGH-Entscheidung Alimanovic zum Ausschluss von Unionsbürgern von sozialen Grundsicherungsleistungen, NVwZ 2015, 1503.

${ }^{7}$ EuGH, Grzelczyk, C-184/99, EU:C:2001:458, Rn. 31.

${ }^{8}$ EuGH, Martínez Sala, C-85/96, EU:C:1998:217.

${ }^{9}$ EuGH, D'Hoop, C-224/98, EU:C:2002:432.

${ }^{10} \mathrm{EuGH}$, MRAX, C-459/99, EU:C:2002:461.

${ }^{11} \mathrm{EuGH}$, Baumbast and $R$, C-413/99, EU:C:2002:493.

${ }^{12}$ E. Spaventa, Earned Citizenship - understanding Union citizenship through its scope, in: D. Kochenov (Hrsg.) EU Citizenship and Federalism: the Role of Rights, CUP 2016 (im Erscheinen). 
tendenziell umfassende Inländerbehandlung“ stehen. ${ }^{13}$ Entscheidende Grundsätze der Rechtsprechung des EuGH wurden sodann in der Unionsbürgerrichtlinie ${ }^{14}$ kodifiziert, welche unter anderem die Bedingungen der Einreise, des Aufenthalts und der Beendigung des Aufenthalts von Unionsbürger*innen und deren Familienmitgliedern regelt und die im Jahr 2004 angenommen und bis zum 30. April $2006^{15}$ von den Mitgliedstaaten in innerstaatliches Recht umzusetzen war. Die Zeit der Kodifizierung der Rechtsprechung des EuGH durch die Unionsbürgerrichtlinie markiert die Phase der Konsolidierung, die durch eine Vertiefung bereits vorhandener sowie die Ausbildung neuer Rechtsprechungsstränge geprägt ist. ${ }^{16}$ Während der in der GrzelczykFormel enthaltene und Artikel 18 AEUV verankerte Grundsatz der gleichen rechtlichen Behandlung von Unionsbürger*innen und Staatsangehörigen die erste und die zweite Phase der Rechtsprechung des EuGH zur Unionsbürgerschaft maßgeblich prägte, kommt den ebenfalls in der Grzelczyk-Formel und Artikel 21 Abs. 1 AEUV erwähnten Bedingungen, Grenzen und Ausnahmen insbesondere in letzter Zeit zunehmende Bedeutung zu. ${ }^{17}$ Werden die ersten beiden Phasen noch als von einem Integrationismus ${ }^{18}$ oder einem, judicial activism ${ }^{\text {"19 }}$ geprägt beschrieben, so wird die gegenwärtige Phase als „doctrinal conservatism“ ${ }^{20}$ bezeichnet.

${ }^{13}$ P. Huber, Unionsbürgerschaft, EuR 2013, 637, 645.

${ }^{14}$ Richtlinie 2004/38/EG des Europäischen Parlaments und des Rates vom 29. April 2004 über das Recht der Unionsbürger und ihrer Familienangehörigen, sich im Hoheitsgebiet der Mitgliedstaaten frei zu bewegen und aufzuhalten, zur Änderung der Verordnung (EWG) Nr. 1612/68 und zur Aufhebung der Richtlinien 64/221/EWG, 68/360/EWG, 72/194/EWG, 73/148/EWG, 75/34/EWG, 75/35/EWG, 90/364/EWG, 90/365/EWG und 93/96/EWG, ABl. 2004 L 158/77 (Unionsbürgerrichtlinie).

${ }^{15}$ Siehe Art. 40 Abs. 1 Unionsbürgerrichtlinie (Fn. 14).

${ }^{16}$ Spaventa, (Fn. 12).

${ }^{17}$ Siehe z.B. EuGH, Dano, C-333/13, EU:C:2014:2358, Rn. 60. Siehe auch D. Thym, Die Rückkehr des „Marktbürgers“ - Zum Ausschluss nichterwerbsfähiger EUBürger von Hartz IV-Leistungen, NJW 2015, 130, 131.

${ }^{18}$ U. Šadl/M. Madsen, Did the Financial Crisis Change European Citizenship Law? An Analysis of Citizenship Rights Adjudication Before and After the Financial Crisis, ELJ 2016, 40, 41.

${ }^{19}$ D. Thym, When Union citizens turn into illegal migrants: the Dano case, EL Rev. 2015, 249, 252.

${ }^{20}$ Thym (Fn. 19), 250. 
Diese konzeptionelle Kehrtwende der Rechtsprechung ist durch eine Abkehr des EuGH von seiner ursprünglichen „Vision“ der Unionsbürgerschaft, hin zu einer minimalistischen Interpretation gekennzeichnet. ${ }^{21}$ Auch wenn angemerkt wurde, dass der Rechtsprechung des EuGH kein einheitliches Konzept der Unionsbürgerschaft zugrunde liegt ${ }^{22}$ und die einzelnen Phasen weder homogen noch linear ${ }^{23}$ sind, so zeichnet sich doch in den letzten Jahren, insbesondere bei Unionsbürger*innen, die auf finanzielle Unterstützung angewiesen oder straffällig geworden sind, eine Rückkehr zu einer stärkeren Betonung der Verantwortlichkeit des Herkunftsstaats für seine eigenen Staatsangehörigen ab. Diese Rückkehr geht mit einer abnehmenden Prüfung und Berücksichtigung der Umstände des Einzelfalles einher, insbesondere den familiären und persönlichen Verbindungen der Unionsbürger*innen im Aufnahmemitgliedstaat. Gleichzeitig droht diese Entwicklung, die verstärkt auf die durch die Staatsangehörigkeit vorgegebenen Bindung abstellt, die durch die Unionsbürgerschaft begünstigte Entwicklung der europäischen Integration, bei der oftmals die von den einzelnen Unionsbürger*innen gewählten Verbindungen zum jeweiligen Aufenthaltsstaat in den Vordergrund treten ${ }^{24} \mathrm{zu}$ untergraben. Dieser Rechtssprechungswechsel ist überdies durch eine zunehmende Bedeutung des Sekundärrechts geprägt, einer damit einhergehenden Verdrängung des Primärrechts, sowie einer Schwächung der Bedeutung der Verhältnismäßigkeitsprüfung. Kennzeichen dieser Wende sind schließlich auch eine bestimmte Interpretation der deutungsoffenen Begriffe der Integration und des sozialen Zusammenhalts durch den EuGH und den Generalanwalt sowie die abnehmende Bedeutung des Begriffes der Solidarität. Der zweite Teil (II.) widmet sich der Verwendung der Begriffe der Integration und des sozialen

21 Spaventa (Fn. 12). Siehe auch S. Coutts, Union citizenship as probationary citizenship: Onuekwere, CML Rev. 2015, 531, 537, 544, der darlegt, dass der EuGH die Unionsbürgerrichtlinie im Lichte einer bestimmten Ideologie und Vision der Unionsbürgerschaft auslegt.

${ }^{22}$ D. Thym, The Elusive Limits of Solidarity: Residence Rights of and Social Benefits for Economically Inactive Union Citizens, CML Rev. 2015, 17, 33.

${ }^{23}$ P. Hilpold, Die Unionsbürgerschaft - Entwicklung und Probleme, EuR 2015, 133, 135.

${ }^{24}$ Spaventa (Fn. 12). Siehe auch Thym (Fn. 22), 34 ff. der zwischen dem "residence model”, das primär auf den Aufenthaltsort abstellt und dem „integration model“, wonach allein der Aufenthalt des Unionsbürgers/der Unionsbürgerin nicht ausreichend ist, unterscheidet. 
Zusammenhalts in der Unionsbürgerrichtlinie und ihrer Interpretation durch den EuGH sowie der Verwendung des Begriffes der Solidarität.

Anschließend findet eine Auseinandersetzung mit verschiedenen, in der Literatur vertretenen, Standpunkten $\mathrm{zu}$ den Auswirkungen der Rechtsprechung des EuGH auf die Umgestaltung der Unionsbürgerschaft statt (III.). Schließlich werden die Folgen der Interpretation der Konzepte der Integration, des sozialen Zusammenhalts und der Solidarität durch den EuGH auf den Gehalt der Unionsbürgerschaft in den Schlussbemerkungen beleuchtet (IV.).

II. Integration, sozialer Zusammenhalt und Solidarität

Die Begriffe der Integration, des sozialen Zusammenhalts und der Solidarität sind interpretationsoffen und auf vielfältige Weise miteinander verknüpft. Auch wenn auf eine Darstellung der verfassungsrechtlichen Dimension dieser Konzepte aus Platzgründen weitgehend verzichtet wird ${ }^{25}$, soll zu Beginn des ersten Abschnitts eine kurze Auseinandersetzung mit zwei unterschiedlichen Deutungsmöglichkeiten des Begriffes der Integration stattfinden. Anschließend beleuchtet der erste Abschnitt die Konzepte der Integration und des sozialen Zusammenhalts beim Ausweisungsschutz und beim Zugang zum Daueraufenthaltsrecht (1.). Der zweite Abschnitt widmet sich sodann dem Begriff der Solidarität beim Zugang zu beitragsunabhängigen Sozialleistungen, indem er auf den (fehlenden) Gebrauch dieses Konzepts durch den EuGH eingeht (2.).

Die Begriffe der Solidarität, des sozialen Zusammenhalts und der Integration, wie sie im AEUV oder in der Grundrechtecharta verwendet werden, sollen nicht oder nur am Rande der Untersuchung Erwähnung finden, da sich der Gerichtshof in seiner Argumentation hierauf nicht bezieht.

${ }^{25}$ Für weitere Diskussion siehe: T. Marshall, Citizenship and Social Class (1950); E. Guild, Does European Citizenship Blur the Borders of Solidarty?, in: Guild/Gortázar Rotaeche/Kostakopulou (Hrsg.): The Reconceptualization of European Union Citizenship (2014), S. 189 F. de Witte, Justice in the EU: The Emergence of Transnational Solidarity (2015). 


\section{Integration und sozialer Zusammenhalt}

Integration ist ein bedeutungsoffener Begriff, der dem Wandel grundlegender gesellschaftlicher Wertvorstellungen unterworfen ist ${ }^{26}$ und der im nationalen ${ }^{27}$ und europäischen Recht ${ }^{28}$ verschiedene Funktionen einnehmen kann. Aus der Perspektive eines rechtsbasierten Ansatzes werden ein sicherer Aufenthaltsstatus und die Gleichbehandlung von Migrant*innen mit Staatsangehörigen des jeweiligen Mitgliedstaates als förderlich für die Integration angesehen. Von einer anderen Perspektive aus betrachtet dient die fehlende Integration von Migrant*innen, die man unter anderem anhand von Sprachkenntnissen, Arbeitsmarktintegration, Kenntnissen der Geschichte, Politik und Gesellschaft des Aufnahmelandes oder mittels anderer Kriterien zu bestimmen versucht, als Grundlage für die Verweigerung des Zugangs oder Aufenthalts oder der Verwehrung bestimmter Rechte im Aufnahmemitgliedstaat. ${ }^{29}$ Beide Perspektiven lassen sich sowohl in Rechtsakten der EU ${ }^{30}$ als auch in der Rechtsprechung des EuGH wiederfinden. ${ }^{31}$

Zudem gibt es vielfältige Formen der Integration, die sich gegenseitig bedingen (können). Eine sprachliche Integration kann eine wirtschaftliche, gesellschaftliche und kulturelle Integration fördern und umgekehrt. Oft wird eine sprachliche Integration sogar als unerlässliche Voraussetzung für eine aktive Teilnahme von Migrant*innen am sozialen, wirtschaftlichen und öffentlichen Leben angesehen. ${ }^{32}$ Aber kann und wenn ja in welchem Umfang die (fehlende) Integration des/r Unionsbürger*in überhaupt berücksichtigt werden? Anders als bei Drittstaatsangehörigen, bei denen die Zuerkennung der Rechtsstellung eines langfristig Aufenthaltsberechtigten

${ }^{26}$ D. Thym, Towards a Contextual Conception of Social Integration in EU Immigration Law. Comments on $P \& S$ and $K \& A$, EJML 2016, 89, $106 \mathrm{f}$.

${ }^{27}$ Siehe zum Begriff der Integration im deutschen Aufenthaltsrecht, J. Eichenhofer, Begriff und Konzept der Integration im Aufenthaltsgesetz, Nomos 2013.

${ }^{28}$ K. Groenendijk, Legal Concepts of Integration in EU Migration Law, EJML 2004, 111,113 .

${ }^{29}$ Zu beiden Perspektiven siehe Thym (Fn. 26), 90, 105 ff; Groenendijk (Fn. 28) 113 ff.

${ }^{30}$ Siehe Groenendijk (Fn. 28), 114 ff., der drei ausgewählte Richtlinien untersucht.

${ }^{31}$ EuGH, Echternach, C-389/87, EU:C:1989:130, Rn. 20 (als Beispiel für die erste Perspektive), EuGH, Dias, C-325/09, EU:C:2011:498, Rn. 64 (als Beispiel für die zweite Perspektive).

${ }^{32}$ Groenendijk (Fn. 28), 114 
gemäß Art. 5 Abs. 2 der Daueraufenthaltsrichtlinie ${ }^{33}$ von der Erfüllung der „Integrationsanforderungen gemäß dem nationalen Recht“ abhängig gemacht werden kann, gibt es eine entsprechende Vorschrift für Unionsbürger*innen nicht. Wie Thym zutreffend feststellt, kennt das Unionsrecht ,spezifische Vorgaben zur sprachlichen und kulturellen Integration“ von Unionsbürger*innen nicht, sodass „die Mitgliedstaaten innerstaatliche Maßnahmen zu verpflichtenden Integrationskursen nach der Einreise sowie notwendigen Sprachkenntnissen bei der Aufenthaltsverfestigung oder beim Ehegattennachzug auf Unionsbürger nicht anwenden dürfen". ${ }^{34}$ Eine entsprechende Integrationsverpflichtung widerspräche nicht nur dem Freizügigkeitsrecht, sondern liefe auch dem supranationalen Integrationsziel zuwider. ${ }^{35}$

Dennoch werden sowohl der Begriff der Integration als auch, unmittelbar damit zusammenhängend, der Begriff des sozialen Zusammenhalts in der (a)) Unionsbürgerrichtlinie verwendet. Beide Begriffe sind für die (b)) Argumentation des Europäischen Gerichtshofs und des Generalanwalts sowohl im Ausweisungsrecht (aa)) als auch im Daueraufenthaltsrecht (bb)) von Bedeutung.

Die nachfolgende Darstellung geht vom Wortlaut der Unionsbürgerrichtlinie aus und wirft einen kritischen Blick auf die Interpretation der Richtlinienbestimmungen durch den EuGH. Dabei sind die beiden soeben dargestellten unterschiedlichen Perspektiven auf den Begriff der Integration - die rechtsbasierte Perspektive, welche die Gleichstellung von Migrant*innen mit Staatsangehörigen als förderlich für das Ziel der Integration betrachtet, sowie die zweite Perspektive, welche die Integration von Migrant*innen zur Voraussetzung für die Gleichstellung mit Staatsangehörigen macht - von zentraler Bedeutung.

Anschließend soll der Frage nachgegangen werden, auf welche Formen der von Unionsbürger*innen zu erbringenden Integration(sleistung) der EuGH abstellt. Dafür wird Coutts' Analyse des vom EuGH verwendeten Integrationsbegriffes herangezogen, welche die Anpassung an die Normen und Werte des Aufnahmemitgliedstaates als die für den EuGH maßgebliche Form der Integration identifiziert (c)). Schließlich erfolgt eine kritische

33 Richtlinie 2003/109/EG des Rates vom 25. November 2003 betreffend die Rechtsstellung der langfristig aufenthaltsberechtigten Drittstaatsangehörigen, AB1. 2004 L 16/44 (Daueraufenthaltsrichtlinie).

${ }^{34}$ D. Thym, Freizügigkeit in Europa als Modell?, EuR 2011, 487, 489 f. Siehe auch Groenendijk (Fn. 28), 116

${ }^{35}$ Groenendijk (Fn. 28), 125; Thym (Fn. 34), 493. 
Auseinandersetzung mit den Folgen eines so verstandenen Integrationsbegriffes (d)).

a) Unionsbürgerrichtlinie

Das Ziel der Integration von Unionsbürger*innen wird im 18. Erwägungsgrund der Präambel der Unionsbürgerrichtlinie genannt, wo es heißt ,(u)m ein wirksames Instrument für die Integration in die Gesellschaft des Aufnahmemitgliedstaats darzustellen, in dem der Unionsbürger seinen Aufenthalt hat, sollte das einmal erlangte Recht auf Daueraufenthalt keinen Bedingungen unterworfen werden". Die Formulierung des 18. Erwägungsgrundes der Präambel unterstreicht, dass das Daueraufenthaltsrecht als ein Instrument verstanden wird, das die Integration von Unionsbürger*innen in die Gesellschaft des Aufnahmemitgliedstaates fördern und ermöglichen soll.

Weiterhin wird im Zusammenhang mit der Ausweisung der Begriff der Integration sowohl im 23. und 24. Erwägungsgrund der Präambel genannt, als auch in Art. 28 Abs. 1 der Unionsbürgerrichtlinie. Letztere Vorschrift enthält verschiedene Kriterien, die ein Mitgliedstaat im Rahmen der Verhältnismäßigkeitsprüfung in die Abwägung einstellen muss, bevor er eine(n) Unionsbürger*in ausweisen kann.

Schließlich ist das Ziel des sozialen Zusammenhalts im 17. Erwägungsgrund der Präambel der Unionsbürgerrichtlinie genannt. Dort heißt es: „(w)enn Unionsbürger, die beschlossen haben, sich dauerhaft in dem Aufnahmemitgliedstaat niederzulassen, das Recht auf Daueraufenthalt erhielten, würde dies ihr Gefühl der Unionsbürgerschaft verstärken und entscheidend zum sozialen Zusammenhalt - einem grundlegenden Ziel der Union - beitragen." Diese Formulierung belegt, dass die Herstellung des sozialen Zusammenhalts als Ziel der Union verstanden wird, welches durch das Mittel der Gewährung des Daueraufenthaltsrechts erreicht werden soll. Sowohl der 17. als auch der 18. Erwägungsgrund der Präambel verdeutlichen, dass die Gewährung des Daueraufenthaltsrechts als ein Instrument zur Erreichung des Ziels der Integration oder der Förderung des sozialen Zusammenhalts verstanden werden. 
b) Interpretation dieser Konzepte durch den EuGH und den Generalanwalt

aa) Im Ausweisungsrecht

Einen anderen Zugang zum Konzept des sozialen Zusammenhalts und der Integration wählte Generalanwalt Bot in der Rechtssache P.I., welche die Ausweisung eines über zehn Jahre in Deutschland lebenden Italieners zum Gegenstand hatte, der wegen sexuellen Missbrauchs eines Kindes, sexueller Nötigung und Vergewaltigung zu einer Freiheitsstrafe von sieben Jahren und sechs Monaten verurteilt wurde. ${ }^{36}$ Aufgrund seiner Aufenthaltsdauer von über zehn Jahren konnte sich P.I. auf den Ausweisungsschutz nach Art. 28 Abs. 3 lit. a der Unionsbürgerrichtlinie berufen. Gemäß Art. 28 Abs. 3 lit. a können Unionsbürger*innen nach über zehnjährigem Aufenthalt nur noch aus zwingenden Gründen der öffentlichen Sicherheit ausgewiesen werden, sodass sich die Frage stellte, ob P.I.'s Verhalten diese Voraussetzung erfüllt. Der Begriff der "zwingenden Gründe der öffentlichen Sicherheit" ist in der Unionsbürgerrichtlinie nicht definiert. In früheren Entscheidungen, die sich jedoch nicht auf Ausweisungen bezogen, hatte der EuGH geurteilt, dass die öffentliche Sicherheit die innere und äußere Sicherheit des Mitgliedstaates betrifft. ${ }^{37}$ Die innere Sicherheit des Staates umfasst die Existenz des Staates, das Funktionieren seiner Einrichtungen und seiner wichtigen öffentlichen Dienste und das Überleben seiner Bevölkerung. ${ }^{38}$ Von dieser Definition ausgehend hatte ein deutsches Gericht dem EuGH bereits in der Rechtssache Tsakouridis die Frage vorgelegt, "ob und inwieweit die mit dem bandenmäßigen Handel mit Betäubungsmitteln verbundene Kriminalität (...) unter den Begriff der ,zwingenden Gründe der öffentlichen Sicherheit" (...) fallen kann" ${ }^{39}$ In der Rechtssache Tsakouridis

\footnotetext{
${ }^{36}$ EuGH, P.I., C-348/09, EU:C:2012:300, Rn. 10.

37 EuGH, Aimé Richardt and Les Accessoires Scientifiques SNC, C-367/89 EU:C:1991:376, Rn. 22 (hinsichtlich Art. 36 AEUV); EuGH, Leifer, C-83/94, EU:C:1995:329, Rn. 26; EuGH, Sirdar, C-273/97, EU:C:1999:523, Rn. 17; EuGH, Albore, C-423/98, EU:C:2000:401, Rn. 18; EuGH, Kreil, C-285/98, EU:C:2000:2, Rn. 17

${ }^{38}$ EuGH, Campus Oil Limited, C-72/83, EU:C:1984:256, Rn. 34.

${ }^{39}$ EuGH, Tsakouridis, C-145/09, EU:C:2010:708, Rn. 39.
} 
antwortete der EuGH, dass ,Ziele wie die Bekämpfung der mit bandenmäßigem Handel mit Betäubungsmitteln verbundenen Kriminalität" nicht vom Begriff der öffentlichen Sicherheit ausgenommen wären. ${ }^{40}$

Damit war aber noch nicht geklärt, ob auch die von P.I. begangenen Straftaten vom Begriff der öffentlichen Sicherheit erfasst sind. In seinem Schlussantrag argumentierte Generalanwalt Bot, dass die von P.I. begangenen Taten nicht unter den Begriff ,zwingende Gründe der öffentlichen Sicherheit" im Sinne von Art. 28 Abs. 3 der Unionsbürgerrichtlinie fallen ${ }^{41}$ sondern vielmehr einen Verstoß gegen die öffentlichen Ordnung darstellen. ${ }^{42}$

Weiterhin führte er aus, dass P.I. ,nicht wirklich integriert war und deshalb nicht in den Genuss des verstärkten Schutzes" gegen Ausweisung, welchen die Unionsbürgerrichtlinie vorsieht, kommen kann. ${ }^{43}$ Zur Begründung verwies er zunächst auf den Zusammenhang zwischen der Ausweisung und der Integration des Unionsbürgers/der Unionsbürgerin, der im 23. und 24. Erwägungsgrund der Unionsbürgerrichtlinie hergestellt wird. ${ }^{44}$ Weiterhin argumentierte er, dass Artikel 28 Abs. 3 der Unionsbürgerrichtlinie eine Vermutung der Integration enthalte, die aber auch widerlegt werden könne. ${ }^{45}$ Sodann brachte er vor, dass „(d)er Unionsgesetzgeber (...) von dem Grundsatz ausgegangen (ist), dass die Dauer des Aufenthalts Aufschluss über eine gewisse Integration in den Aufnahmemitgliedstaat gibt. Nach einem Zeitraum von zehn Jahren wird vermutet, dass zwischen dem Unionsbürger (...) und dem genannten Staat so enge Bindungen bestehen, dass sie diesem Bürger das Gefühl geben können, Teil der Gesellschaft des betreffenden Staates zu sein, wobei dies (...) zum sozialen Zusammenhalt, einem grundlegenden Ziel der Union, beitragen soll““. ${ }^{46}$ Die Integration des Unionsbürgers/der Unionsbürgerin beruhe, so Bot, nicht nur auf räumlichen und zeitlichen Umständen, sondern auch qualitativen Faktoren. P.I.'s Verhalten zeuge vom völligen Fehlen

${ }^{40}$ EuGH, Tsakouridis (Fn. 39), Rn. 45.

${ }^{41}$ GA Bot, P.I., C-348/09, EU:C:2012:123, Rn. 24-47.

${ }^{42}$ GA Bot, P.I. (Fn. 41), Rn. 60.

${ }^{43}$ GA Bot, P.I. (Fn. 41), Rn. 49.

${ }^{44}$ GA Bot, P.I. (Fn. 41), Rn. 53, 54.

${ }^{45}$ GA Bot, P.I. (Fn. 41), Rn. 56.

${ }^{46}$ GA Bot, P.I. (Fn. 41), Rn. 57. 
eines Willens, sich in die Gesellschaft zu integrieren. ${ }^{47} \mathrm{Im}$ Ergebnis argumentierte Generalanwalt Bot, dass sich P.I. nicht auf den Ausweisungsschutz nach Artikel 28 Abs. 3 (sowie Abs. 2) der Unionsbürgerrichtlinie berufen könne, wenn erwiesen sei, dass er dieses Recht aus einem gesetzwidrigen Verhalten herleite, das eine schwerwiegende Störung der öffentlichen Ordnung des Aufnahmemitgliedstaats darstelle. ${ }^{48}$

Der Gerichtshof folgte der Argumentation des Generalanwalts nicht, sondern stellte es den Mitgliedstaaten frei, die von P.I. begangenen Straftaten, bzw. Straftaten die in Art. 83 Abs. 1 Unterabs. 2 AEUV aufgeführt sind, als geeignet anzusehen, die Ruhe und die physische Sicherheit der Bevölkerung unmittelbar zu bedrohen, mit der Folge, dass sie unter den Begriff der zwingenden Gründe der öffentlichen Sicherheit fallen können. ${ }^{49}$

Nicht nur der vom Gerichtshof beschrittene Weg ${ }^{50}$, sondern auch die von Generalanwalt Bot gewählte Argumentation ist problematisch, weil sie den durch die Unionsbürgerrichtlinie gewährten Schutz vor Ausweisung untergräbt.

Hinsichtlich der Argumentation des Generalanwalts ist hervorzuheben, dass der 23. Erwägungsgrund der Präambel der Unionsbürgerrichtlinie bestimmt, dass ,,(d)ie Ausweisung von Unionsbürgern (...) aus Gründen der öffentlichen Ordnung oder Sicherheit (...) eine Maßnahme (ist), die Personen, die ihre Rechte und Freiheiten aus dem Vertrag in Anspruch genommen haben und vollständig in den Aufnahmemitgliedstaat integriert sind, sehr schaden kann". Dies verdeutlicht, dass auch Unionsbürger*innen, die eine Gefahr für die öffentliche Ordnung oder sogar für die öffentliche Sicherheit darstellen, in den jeweiligen Mitgliedstaat integriert sein können.

${ }^{47}$ GA Bot, P.I. (Fn. 41), Rn. 60.

${ }^{48}$ GA Bot, P.I. (Fn. 41), Rn. 64.

${ }^{49}$ EuGH, P.I. (Fn. 36), Rn. 33.

${ }^{50}$ Kritisch: G. Anagnostaras, Enhanced protection of EU nationals against expulsion and the concept of internal public security: Comment on the PI case, EL Rev. 2012, $627,630,634 ;$ D. Kochenov, B. Pirker, Deporting the Citizens within the European Union: A Counter-Intuitive Trend in Case C-348/09, P.I. v Oberbürgermeisterin der Stadt Remscheid, Columbia Journal of European Law 2013, 369, 388; D. Kostakopoulou, N. Ferreira, Testing Liberal Norms: The Public Policy and Public Security Derogations and the Cracks in European Union Citizenship, Columbia Journal of European Law 2014, 167, $174 \mathrm{f}$. 
Die Begehung selbst schwerster Straftaten und die Integration im Aufenthaltsmitgliedstaat stehen demnach nicht zwingend in einem Ausschließlichkeitsverhältnis.

Jenseits der Präambel wird der Begriff der Integration nur in Art. 28 Abs. 1 der Unionsbürgerrichtlinie verwendet, der eine nicht abschließende Liste der Kriterien enthält, die ein Mitgliedstaat in die Abwägung einstellen muss, bevor er eine(n) Unionsbürger*in ausweisen kann. In Art. 28 Abs. 1 sind neben der Dauer des Aufenthalts und der familiären und wirtschaftlichen Lage auch die soziale und kulturelle Integration im Aufnahmemitgliedstaat genannt. Die Integration von Unionsbürger*innen im Aufenthaltsstaat ist mithin ein Kriterium, das vor einer Ausweisung berücksichtigt werden muss und einer Ausweisung entgegenstehen kann. ${ }^{51}$ Die Integration stellt aber keine Voraussetzung dar, die erfüllt werden muss, um überhaupt in den Genuss des Ausweisungsschutzes zu kommen.

Die vom Generalanwalt aufgestellte These der widerlegbaren Vermutung der Integration stellt eine Einschränkung des Ausweisungsschutzes dar, die ihre Stütze weder im Wortlaut ${ }^{52}$, dem Sinn und Zweck, der Systematik, noch der Entstehungsgeschichte der Vorschrift findet. Schließlich enthält die Unionsbürgerrichtlinie keine qualitativen Anforderungen, wie den Nachweis der Integration im Aufenthaltsmitgliedstaat. Die Gewährung des nach Art. 28 abgestuften Ausweisungsschutzes ist nach dem Wortlaut der Richtlinie nur von einem temporalen und territorialen Faktor abhängig.

Die durch den Generalanwalt vorgenommene Umkehrung der Bedeutung der Integration von einem Kriterium bei der Abwägung, die einer Ausweisungsentscheidung vorauszugehen hat, hin zu einem Kriterium, welches der/die Unionsbürger*in erfüllen muss, um sich auf den Ausweisungsschutz berufen zu können, hätte die rückwirkende Einschränkung des durch die Unionsbürgerrichtlinie gewährten Ausweisungsschutzes ermöglicht. Durch das Hineinlesen neuer Voraussetzungen in die Vorschrift des Art. 28 der Unionsbürgerrichtlinie wäre der Schutz der Unionsbürger*innen vor Ausweisungen verkürzt

${ }^{51}$ Siehe auch L. Azoulai, S. Coutts, Restricting Union citizens' residence rights on ground of public security, Where Union citizenship and the AFSJ meet: P.I., CML Rev. 2013, 553, 562. C. Murphy, Immigration, Integration and the Law (2016), S. 196. Murphy bezeichnet die Integration ausdrücklich als Schranke für die Ausweisung von Unionsbürger*innen.

${ }^{52}$ Siehe Anagnostaras (Fn. 50), 637. 
worden. Weiterhin ist nicht klar, in welchen Fällen davon ausgegangen werden könnte, dass der/die Unionsbürger*in ausreichend integriert ist, da, wie soeben festgestellt, die Begehung von Straftaten und eine Integration im Aufenthaltsmitgliedstaat sich nicht notwendig gegenseitig ausschließen müssen. Die Argumentation des Generalanwalts wurde jedoch in einer späteren Entscheidung teilweise durch den EuGH aufgegriffen, der in der Rechtssache M.G. ausführte, dass ,,der Grad der Integration der Betroffenen die wesentliche Grundlage sowohl für das Daueraufenthaltsrecht als auch für die Regelung zum Schutz vor Ausweisungsmaßnahmen (...) bildet" .53

\section{bb) Im Daueraufenthaltsrecht}

Der von Generalanwalt Bot in der Rechtssache P.I. gewählte Zugang, nicht lediglich auf temporale und territoriale Faktoren abzustellen, sondern zusätzlich noch „qualitative Faktoren“ einzubeziehen, war vom EuGH zuvor schon im Bereich des Daueraufenthaltsrechts (Art. 16 der Unionsbürgerrichtlinie) entwickelt worden. Bereits im Jahr 2011 wies der Gerichtshof in der Rechtssache Dias auf den Integrationsgedanken hin, ,der dem Erwerb des Daueraufenthaltsrechts nach Art. 16 Abs. 1 der Richtlinie 2004/38 zugrunde liegt“ und der „nicht nur auf territoriale und zeitliche Umstände, sondern auch auf qualitative Elemente im Zusammenhang mit dem Grad der Integration im Aufnahmemitgliedstaat abstellt". ${ }^{54}$ Auf den sozialen Zusammenhalt nahm der EuGH hingegen nicht Bezug.

Anders jedoch in der Rechtssache Onuekwere, in der es um den drittstaatsangehörigen Familienangehörigen einer Unionsbürgerin ging. Dort wiederholte der EuGH zunächst den im 17. Erwägungsgrund enthaltenen Gedanken, dass das Recht auf Daueraufenthalt entscheidend zum sozialen Zusammenhalt beitrage, fügte dann aber, wie zuvor schon in der Rechtssache Lassal ${ }^{55}$ aus, dass der Unionsgesetzgeber die Erlangung eines Daueraufenthaltsrechts nach Art. 16 der Unionsbürgerrichtlinie „,von der Integration des Unionsbürgers in den Aufnahmemitgliedstaat abhängig gemacht hat". ${ }^{56}$ Obwohl der Wortlaut des Art. 16 Abs. 1. S. 1 der

\footnotetext{
${ }^{53}$ EuGH, M.G., C-400/12, EU:C:2014:9, Rn. 32.

${ }^{54}$ EuGH, Dias (Fn. 31), Rn. 64.

${ }^{55}$ EuGH, Lassal, C-162/09, EU:C:2010:592, Rn. 37.

${ }^{56}$ EuGH, Onuekwere, C-378/12, EU:C:2014:13, Rn. 24.
} 
Unionsbürgerrichtlinie bestimmt, dass ,(j)eder Unionsbürger, der sich rechtmäßig fünf Jahre lang ununterbrochen im Aufnahmemitgliedstaat aufgehalten hat, (...) das Recht (hat), sich dort auf Dauer aufzuhalten" kein Integrationserfordernis statuiert, wiederholte der EuGH sodann seine bereits in Dias getroffene Aussage, dass Art. 16 Abs. 1 der Unionsbürgerrichtlinie auch auf qualitative Elemente im Zusammenhang mit dem Grad der Integration im Aufnahmemitgliedstaat verweist. ${ }^{57}$ Weiterhin führt er aus, dass eine „Verpflichtung zur Integration, (...) dem Erwerb des Daueraufenthaltsrechts zugrunde liegt ${ }^{\prime} .{ }^{58}$ Diese Aussage überrascht vor dem Hintergrund des 18. Erwägungsgrunds der Präambel der Unionsbürgerrichtlinie, der das Daueraufenthaltsrecht als Instrument für die Integration von Unionsbürger*innen in die Gesellschaft des Aufnahmemitgliedstaates bezeichnet. Indem der Gerichtshof das Daueraufenthaltsrecht von der Integration in die Gesellschaft und dem sozialen Zusammenhalt abhängig macht, scheint er das Ziel (Herstellung des sozialen Zusammenhalts und Integration von Unionsbürger*innen) und das Mittel (Gewährung des Daueraufenthaltsrechts) zu vertauschen. ${ }^{59}$

Es ist bemerkenswert, dass der EuGH neben den in Art. 16 der Unionsbürgerrichtlinie aufgestellten Kriterien der Dauer des rechtmäßigen Aufenthalts und des Aufenthaltsortes, zusätzlich noch qualitative Kriterien verlangt und somit eine weitere Hürde für die Berufung auf das Daueraufenthaltsrecht aufstellt. Das Daueraufenthaltsrecht hat eine zentrale Stellung zum einen für das soeben erörterte Ausweisungsrecht, da Art. 28 Abs. 2 der Unionsbürgerrichtlinie einen verstärkten Ausweisungsschutz für Inhaber des Daueraufenthaltsrechts dahingehend vorsieht, dass sie nur noch aus schwerwiegenden Gründen der öffentlichen Sicherheit oder Ordnung ausgewiesen werden dürfen. Zum anderen hat das Daueraufenthaltsrecht besondere Bedeutung für den Zugang zu beitragsunabhängigen Sozialleistungen, da Art. 16 Abs. 1 S. 2 der Unionsbürgerrichtlinie

${ }^{57}$ EuGH, Onuekwere (Fn. 56), Rn. 25.

${ }^{58}$ EuGH, Onuekwere (Fn. 56), Rn. 30.

${ }^{59}$ Siehe auch L. Azoulai der hervorhebt, dass der Gerichtshof die Bedeutung des Konzepts des sozialen Zusammenhalts ändert, indem er den Erwerb des Daueraufenthaltsrechts von der Integration des Unionsbürgers/der Unionsbürgerin im Aufenthaltsmitgliedstaat abhängig macht, L. Azoulai, The (Mis)Construction of the European Individual: Two Essays on Union Citizenship Law, EUI Department of Law Research Paper No. 2014/14, S. 16. 
bestimmt, dass „(d)ieses Recht (...) nicht an die Voraussetzungen des Kapitels III geknüpft (ist)“ und damit der/die Unionsbürger*in beispielsweise nicht mehr ausreichende finanzielle Mittel nachweisen muss.

Die dem Urteil des Gerichtshofes und den Schlussanträgen des Generalanwalts zugrunde liegende Logik kommt in der Aussage des Generalanwalts deutlich zum Ausdruck, wenn dieser ausführt, dass das Daueraufenthaltsrecht auf dem Gedanken beruhe, „dass eine echte Integration gewissermaßen belohnt werden“ 60 müsse. Das Daueraufenthaltsrecht wird somit zu einem Instrument der Belohnung für eine erfolgreiche Integration(sleistung) gemacht, bei der die einzelnen Unionsbürger*innen vorleistungspflichtig sind. Diese Herangehensweise lässt sich jedoch nicht leicht mit dem in der Unionsbürgerrichtlinie verwendeten Begriff der Integration vereinbaren.

Die Bezugnahmen auf den Begriff der Integration in der Unionsbürgerrichtlinie sind, wie soeben dargelegt, vor allem durch die rechtsbasierte Perspektive geprägt, ${ }^{61}$ bei der die Gleichstellung von Unionsbürger*innen und Staatsangehörigen des jeweiligen Mitgliedstaats im Vordergrund steht, um die Integration von Unionsbürger*innen zu fördern. Der Zugriff des EuGH auf den Begriff der Integration und des sozialen Zusammenhalts lässt hingegen einen anderen Schwerpunkt erkennen, der eher dem Verständnis der zweiten Perspektive entspricht, bei der eine wie auch immer definierte Integration zur Voraussetzung für die Inanspruchnahme von Rechten gemacht wird. Während Aufenthaltssicherheit und Gleichberechtigung nicht nur bei Unionsbürger*innen, sondern auch bei Drittstaatsangehörigen grundlegende Mechanismen sind, um die Integration des Individuums zu ermöglichen und seine Inklusion zu fördern ${ }^{62}$, wird Integration nunmehr durch die Rechtsprechung des Gerichtshofs zur Bedingung für den Erwerb des Daueraufenthaltsrechts nach der Unionsbürgerrichtlinie gemacht. ${ }^{63}$ Parallel zu der eingangs erwähnten zweigliedrigen Grzelczyk-Formel, weist

${ }^{60}$ GA Bot, Onuekwere, C-378/12, EU:C:2013:640, Rn. 47.

${ }^{61}$ Siehe auch Groenendijk (Fn. 28), 125.

${ }^{62} \mathrm{~K}$. Groenendijk, Long-term immigrants and the Council of Europe, in: Guild/ Minderhoud (Hrsg.): Security of Residence and Expulsion (2001), S. 7; D. Acosta, The Long-Term Resident Status as a Subsidiary Form of EU Citizenship, An Analysis of Directive 2003/109 (2011), S. 138 (hinsichtlich der Daueraufenthaltsrichtlinie).

${ }^{63}$ Coutts (Fn. 21), 537, 538. 
auch der Begriff der Integration mehrere Funktionen und Deutungsmöglichkeiten auf. Es gibt jedoch auch einen entscheidenden Unterschied zwischen der zweigliedrigen Grzelczyk-Formel und den unterschiedlichen Deutungsmöglichkeiten des Begriffes der Integration. Bei der durch den EuGH geschaffenen und im Primärrecht verankerten Grzelczyk-Formel, stehen zwei Elemente gleichberechtigt nebeneinander. Das Element der Gleichbehandlung von Unionsbürger*innen mit Staatsangehörigen des jeweiligen Aufenthaltsstaates ist ebenso in der Grzelczyk-Formel verankert wie die Beschränkungen und Bedingungen, die mit dem Unionsbürgerstatus einhergehen können. Diese Gleichberechtigung beider Elemente gibt es bei der Verwendung des Begriffes der Integration in der Unionsbürgerrichtlinie hingegen nicht. Die Unionsbürgerrichtlinie betrachtet die Integration als ein Ziel, das durch Gleichstellung von Unionsbürger*innen und Staatangehörigen des jeweiligen Mitgliedstaates erreicht werden soll. Die zweite Perspektive, welche den Begriff der Integration als Beschränkungsmechanismus verwendet, wird erst durch den EuGH beziehungsweise durch den Generalanwalt eröffnet. Durch diese Herangehensweise an den Begriff der Integration und, damit zusammenhängend des sozialen Zusammenhalts, leistet der EuGH einen entscheidenden Beitrag zur Umgestaltung der Unionsbürgerschaft.

\section{c) Maßgebliche Form(en) der Integration}

Auf welche Form(en) der Integration stellen nun aber der Gerichtshof und der Generalanwalt ab? Coutts identifiziert die Anpassung an die Werte und Sozialnormen des Aufnahmelandes als die nach der Rechtsprechung des EuGH für das Daueraufenthaltsrecht und das Ausweisungsrecht entscheidende Form der Integration. Er argumentiert, dass der EuGH und der Generalanwalt die Unionsbürgerschaft als einen Status der Integration betrachten, der die Einhaltung und Anpassung an die Werte und Sozialnormen einer bestimmten Gemeinschaft erfordere. ${ }^{64}$ Er vertritt den Standpunkt, dass für den EuGH und den Generalanwalt nicht die durch die Inhaftierung hervorgerufene physische und soziale Trennung des

\footnotetext{
${ }^{64}$ Coutts (Fn. 21), 539.
} 
Individuums von der Gemeinschaft und die damit verbundene Hemmung der Integration (,internal exile“) im Vordergrund stehen, sondern vielmehr das Unrecht, das gegen die Gesellschaft begangen wurde. ${ }^{65}$ Generalanwalt und Gerichtshof sähen die Verurteilung und die Inhaftierung als Stellvertreter oder Konsequenz der Missachtung oder Zurückweisung gesellschaftlicher Werte an, welche die Integration unterbreche oder annulliere oder sogar eine komplette Abwesenheit von Integration des jeweiligen Individuums offenbare. ${ }^{66}$ Weiterhin führt er aus, dass in Tsakouridis und P.I. die Ausweisung als eine Form der Risikokontrolle und Schadensreduzierung diene und als eine Maßnahme, die dazu bestimmt ist, die Werte der Gesellschaft des Aufnahmemitgliedstaates durchzusetzen und $\mathrm{zu}$ schützen. Während diese Begründung in den beiden Ausweisungsentscheidungen nur implizit zum Ausdruck komme, tritt sie, so Coutts, in Onuekwere, deutlich hervor. In Onuekwere werde die Darstellung des Strafrechts als die Reflektion sozialer Normen und Werte der „Gastgesellschaft“ deutlich ${ }^{67}$, wenn der EuGH ausführt, dass „,der Betroffene die von der Gesellschaft des Aufnahmemitgliedstaats in dessen Strafrecht zum Ausdruck gebrachten Werte nicht beachtet" ${ }^{68}$

d) Integration als Anpassung an die Werte und Sozialnormen des Aufnahmemitgliedstaates?

Die Anpassung an die Werte und Sozialnormen des Aufnahmemitgliedstaates als die für den Erwerb des Daueraufenthaltsrechts und den Schutz vor Ausweisung maßgebliche Form der Integration wirft jedoch für das Daueraufenthaltsrecht sowie das Ausweisungsrecht Fragen auf.

Im Kontext des Daueraufenthaltsrechts verdrängt die Gleichstellung von Einhaltung der Rechtsnormen mit erfolgreicher Integration andere Formen der Integration, wie zum Beispiel eine sprachliche oder wirtschaftliche Integration. Abgesehen von dem Umstand, dass Art. 16 der Unionsbürgerrichtlinie das Kriterium der Integration nicht aufführt, ist auch nicht ersichtlich, warum andere Formen der Integration weniger oder

${ }^{65}$ Coutts (Fn. 21), 540.

${ }^{66}$ Coutts (Fn. 21), 541.

${ }^{67}$ Coutts (Fn. 21), 543.

${ }^{68} \mathrm{EuGH}$, Onuekwere (Fn. 56), Rn. 26. 
vielleicht sogar gar keine Bedeutung haben sollen, wenn man den Erwerb des Daueraufenthaltsrechts schon von einer Integration abhängig macht.

Im Ausweisungsrecht wirft das Verständnis von Integration als Anpassung an die Normen und Werte des Aufnahmemitgliedstaats zwei zentrale Fragen auf. Zum einen ist dieses Verständnis von Integration nicht unbedingt leicht mit dem im Kontext des Ausweisungsrechts aufgezeigten Umstand zu vereinbaren, dass Unionsbürger*innen, welche die Strafnormen des Aufnahmemitgliedstaates verletzen und eine Gefahr für die öffentliche Ordnung oder sogar für die öffentliche Sicherheit darstellen, zugleich auch im jeweiligen Mitgliedstaat integriert sein können. Bei den Ausführungen zum Ausweisungsrecht wurde aufgezeigt, dass die Begehung von Straftaten und die Integration im Aufnahmemitgliedstaat nicht zwingend in einem Ausschließlichkeitsverhältnis stehen wie sich auch aus dem 23. Erwägungsgrund der Präambel der Unionsbürgerrichtlinie ergibt. Dieser Erwägungsgrund weist darauf hin, dass eine Ausweisung denjenigen Personen, die vollständig im Aufnahmemitgliedstaat integriert sind, sehr schaden kann.

Außerdem ist bei einer Gleichstellung von Integration mit der Achtung von Werten und Sozialnormen des Aufnahmemitgliedstaates unklar, ob die in Art. 28 Abs. 1 der Unionsbürgerrichtlinie erwähnte Prüfung der sozialen und kulturellen Integration im Aufnahmemitgliedstaat, die einer Ausweisung vorauszugehen hat, noch einen eigenständigen Anwendungsbereich hätte.

Zum anderen führt die Betonung der Werte und Normen des jeweiligen Mitgliedstaates dazu, dass der Blick auf die europäische Ebene verstellt wird. Sowohl in Tsakouridis als auch in P.I. konnten die Unionsbürger einen über zehnjährigen Aufenthalt im Aufnahmemitgliedstaat vorweisen, sodass die Ausweisung nur aus zwingenden Gründen der öffentlichen Sicherheit verfügt werden konnte, wobei die öffentliche Sicherheit als die innere und äußere Sicherheit des Staates definiert wird. In P.I. urteilte der EuGH, ,dass es den Mitgliedstaaten freisteht, Straftaten wie die in Art. 83 Abs. 1 Unterabs. 2 AEUV angeführten als besonders schwere Beeinträchtigung eines grundlegenden gesellschaftlichen Interesses anzusehen, die geeignet ist, die Ruhe und die physische Sicherheit der Bevölkerung unmittelbar zu bedrohen, und die damit unter den Begriff der 
zwingenden Gründe der öffentlichen Sicherheit fallen kann““ ${ }^{69}$ Während Coutts in dieser Formulierung auch eine Maßnahme erblickt, die dazu bestimmt ist, die Werte der Gesellschaft des Aufnahmemitgliedstaates durchzusetzen und zu schützen ${ }^{70}$, kommt durch die Berufung auf Art. 83 Abs. 1 AEUV noch ein anderer Aspekt zum Vorschein. Einige Randnummern zuvor hatte der EuGH ausgeführt, dass „Art. 83 Abs. 1 AEUV (...) zu den Bereichen besonders schwerer Kriminalität (gehört), die eine grenzüberschreitende Dimension haben und für die ein Tätigwerden des Unionsgesetzgebers vorgesehen ist" ${ }^{71}$ Anders als in der Rechtssache Onuekwere, in der die Missachtung der Normen des Aufenthaltsstaates im Vordergrund steht, verweist der EuGH in P.I. auf Straftaten, die eine grenzüberschreitende Dimension haben und damit neben der Gesellschaft des Aufnahmemitgliedstaates auch andere Gesellschaften betreffen. Die von P.I. begangenen Straftaten (sexueller Missbrauch eines Kindes, sexuelle Nötigung und Vergewaltigung) stellen zweifelsfrei eine Missachtung der Normen des Aufnahmemitgliedstaates dar, ebenso wie der Werte und Normen des Herkunftsmitgliedstaates und der Union. Die Fokussierung auf die Werte des Aufnahmemitgliedstaates ist logisch, geht es doch um die Ausweisung aus diesem Staat oder, wie in der Rechtssache Onuekwere, um die Verwehrung des Daueraufenthaltsrechts in diesem Staat. Sie verstellt aber den Blick auf den Umstand, dass die von P.I. begangenen Straftaten eine Missachtung der Werte der Union und ihrer Mitgliedstaaten darstellen und davon zeugen, dass der betreffende Unionsbürger in keinem Mitgliedstaat der Union ,,integriert“, ist, wenn man Integration als Achtung der Werte und Normen definiert. An dieser Stelle rückt die eingangs erwähnte Entwicklung, verstärkt auf die durch die Staatsangehörigkeit vorgegebenen Bindungen abzustellen, in den Vordergrund. Ein Mitgliedstaat, im Falle von P.I. Italien, muss einen Staatsangehörigen, der seit über zehn Jahren in einem anderen Mitgliedstaat (Deutschland) lebt und dessen Geschwister und Mutter ebenfalls teilweise in Deutschland leben ${ }^{72}$, zurücknehmen. Eine derartige Pflicht des Staates der Staatsangehörigkeit, die zweifelsohne ihre Grundlage in der Unionsbürgerrichtlinie findet, wirft nicht nur Fragen des geeigneten Ortes der Resozialisierung oder der Reintegration von Unionsbürger*innen auf,

\footnotetext{
${ }^{69}$ EuGH, P.I. (Fn. 36), Rn. 33.

${ }^{70}$ S. Coutts (Fn. 21), 541.

${ }^{71}$ EuGH, P.I. (Fn. 36), Rn. 25.

${ }^{72}$ EuGH, P.I. (Fn. 36), Rn. 9.
} 
sondern stellt damit auch die Solidarität der Mitgliedstaaten untereinander auf den Prüfstand.

\section{Solidarität und Integration bei der Gewährung von beitragsunabhängigen Sozialleistungen}

Sowohl in Fällen des Ausweisungsschutzes als auch des Daueraufenthaltsrechts, die beide primär auf quantitativen (temporalen und territorialen) Kriterien basieren, verlangen der EuGH bzw. der Generalanwalt zusätzlich eine qualitative Komponente, indem sie die Integration des Unionsbürgers/der Unionsbürgerin in den Blick nehmen. Durch diese Herangehensweise reduzieren sie den Anwendungsbereich des Daueraufenthaltsrechts, was, wie bereits erwähnt, sowohl Implikationen für den Ausweisungsschutz als auch für die Gewährung von beitragsunabhängigen Sozialleistungen hat.

Zentrale Kriterien beim Zugang $\mathrm{zu}$ beitragsunabhängigen Sozialleistungen scheinen in der neueren Rechtsprechung des EuGH vor allem die wirtschaftliche Integration oder die wirtschaftliche Unabhängigkeit des Unionsbürgers/der Unionsbürgerin zu sein.

Der Grundsatz der Solidarität, der ausweislich des Art. 2 EUV $^{73}$ ein gemeinsamer Wert ist, auf den sich die Union gründet und dem in der Grundrechtecharta ein eigenes Kapitel gewidmet ist, nicht zuletzt um dem wirtschaftlichen Konzept des Binnenmarktes eine soziale Dimension zu geben ${ }^{74}$, tritt dabei zunehmend in den Hintergrund. Von den unterschiedlichen Erscheinungsformen der Solidarität in der europäischen Rechtsordnung 75 soll an dieser Stelle nur die Solidarität unter Unionsbürger*innen in den Blick genommen werden, wobei Solidarität hier primär als eine finanzielle Beistandspflicht verstanden wird. Ebenso wie beim Ausweisungsrecht und Daueraufenthaltsrecht ist auch beim Zugang

${ }^{73}$ Vertrag über die Europäische Union, AB1. 2012, C 326/01 (EUV).

${ }^{74}$ I. Domurath, The Three Dimensions of Solidarity in the EU Legal Order: Limits of the Judicial and Legal Approach, Journal of European Integration 2013, 459, 460.

${ }^{75}$ Für einen Überblick siehe Domurath (Fn.74), 459 ff. und A. Farahat, Solidarität und Inklusion - Umstrittene Dimensionen der Unionsbürgerschaft-, DÖV 2016, 45, 53. 
zu beitragsunabhängigen Sozialleistungen im Aufnahmemitgliedstaat eine Verschiebung der Schwerpunkte in der Rechtsprechung des EuGH zu beobachten.

Während der EuGH noch im Jahr 2013 in der Rechtssache Brey auf die in der Grzelczyk-Entscheidung verwendete Formulierung der „finanzielle(n) Solidarität der Staatsangehörigen des Aufnahmemitgliedstaats mit denen der anderen Mitgliedstaaten" ${ }^{\text {"76 }}$ Bezug nahm, finden sich entsprechende Verweise auf eine finanzielle Solidarität weder in den späteren Entscheidung in den Rechtssachen Dano, Alimanovic und Peña-García wieder noch wird der Begriff der „Solidarität“ in den Urteilsbegründungen verwendet. Auch wenn Hilpold ausführt, dass die Dano-Entscheidung ,statt einer , grenzenlosen Solidarität ${ }^{6}$, wie das ,Brey“Kriterium nahezulegen scheint", eine gewisse Solidarität einzufordern scheint, ${ }^{77}$ so wird jedoch nicht ersichtlich, worin diese Solidarität im Fall von Frau Dano liegen soll.

Weiterhin ändert sich der Fokus von einer Einzelfallbetrachtung und Verhältnismäßigkeitsprüfung in der Brey-Entscheidung ${ }^{78}$, hin zu einer Prüfung der überwiegend wirtschaftlichen Faktoren des Art. 7 Abs. 1 der Unionsbürgerrichtlinie in der Rechtssache Dano $^{79}$ ohne Berücksichtigung der familiären Umstände und ohne dass eine Verhältnismäßigkeitsprüfung stattfindet. ${ }^{80}$ Auch wenn der Gerichtshof in Dano das Erfordernis einer Prüfung der wirtschaftlichen Situation jedes Betroffenen aufstellt, um beurteilen zu können, „ob ein Unionsbürger über ausreichende Existenzmittel verfügt, um ein Aufenthaltsrecht nach Art. 7 Abs. 1

${ }^{76}$ EuGH, Brey, C-140/12, EU:C:2013:565, Rn. 72.

${ }^{77}$ Hilpold (Fn. 23), 143.

${ }^{78}$ EuGH, Brey (Fn. 76), Rn. 75.

${ }^{79} \mathrm{EuGH}$, Dano (Fn. 17), Rn. 78.

${ }^{80}$ Siehe N. Nic Shuibhne, Limits rising, duties ascending: The changing legal shape of Union citizenship, CML Rev. 2015, 889, 913; F. Wollenschläger, Keine Sozialleistungen für nichterwerbstätige Unionsbürger? Zur begrenzten Tragweite des Urteils des EuGH in der Rechtssache Dano vom 11.11.2014, NVwZ 2014, 1628, 1630, der auf das Fehlen der Verhältnismäßigkeitsprüfung hinweist. Für Kommentare und Interpretation des Urteils siehe: H. Verschueren, Free Movement of EU citizens, Including for the Poor?, Maastricht Journal of European and Comparative Law 2015, 10, 27; H. Verschueren, Preventing "Benefit-Tourism" in the EU: A Narrow or Broad Interpretation of the Possibilities Offered by the ECJ in Dano? CML Rev. 2015, 363, 373. 
Buchst. b der Unionsbürgerrichtlinie in Anspruch nehmen zu können" ${ }^{\text {"81 }}$ und damit eine Prüfung der Einzelfallumstände vornimmt, ${ }^{82}$ so ist diese Prüfung doch auf die finanzielle Situation des Individuums beschränkt. Die Tatsache, dass Frau Dano und ihr Sohn Florin in der Wohnung der Schwester von Frau Dano leben, die sie mit Naturalien versorgt, ${ }^{83}$ findet hingegen keine Berücksichtigung.

Das Fehlen der Berücksichtigung der Umstände des Einzelfalles in der Rechtssache Dano setzt sich in Alimanovic fort. Der EuGH führt dazu aus, dass die Berücksichtigung der Umstände des Einzelfalles entbehrlich sei, da die Unionsbürgerrichtlinie, die „ein abgestuftes System für die Aufrechterhaltung der Erwerbstätigeneigenschaft schafft (...) nämlich selbst verschiedene Faktoren, die die jeweiligen persönlichen Umstände der eine Sozialleistung beantragenden Person kennzeichnen, insbesondere die Dauer der Ausübung einer Erwerbstätigkeit" berücksichtigt. ${ }^{84}$ Die gleiche Argumentation findet sich in der Rechtssache Peña-García wieder, in der es, zumindest bei Herrn Peña-Cuevas, um einen unter drei Monate dauernden Aufenthalt ging, sodass Art. 6 der Unionsbürgerrichtlinie einschlägig war. ${ }^{85}$ Der EuGH urteilte, dass wenn eine Prüfung der Umstände des Einzelfalles in der Rechtssache Alimanovic, also „bei einem Arbeit suchenden Unionsbürger, der nicht mehr den Status eines Erwerbstätigen hat", nicht erforderlich ist, dies erst recht bei Personen gelten müsse, die einen Aufenthalt von unter drei Monaten haben und nicht erwerbstätig sind. ${ }^{86}$ Der Umstand, dass zwei Kinder der Peña-García Familie die Schule in Deutschland besuchen und dass die Familie bei der Mutter von Frau García-Nieto wohnte ${ }^{87}$, finden hingegen keine Berücksichtigung.

Die Argumentation in Alimanovic und Peña-García begegnet mehreren Bedenken. ${ }^{88}$ Auch wenn das stufenweise System und die jeweiligen

${ }^{81}$ EuGH, Dano (Fn. 17), Rn. 80.

${ }^{82}$ Thym (Fn. 22), 31 sieht darin einen Beleg für die individuelle Prüfung durch den Gerichtshof; siehe ebenso Hilpold (Fn. 23), 143, der betont, dass die Prüfung zwar einzelfallbezogen bleibt, doch „hier nicht die äußerst anspruchsvollen Kriterien, die der EuGH in „Brey“ für den Entzug des Aufenthaltsrechts entwickelt hat“ gelten.

${ }^{83}$ EuGH, Dano (Fn. 17), Rn. 37.

${ }^{84}$ EuGH, Alimanovic, C-67/14, EU:C:2015:597, Rn. 60.

${ }^{85}$ EuGH, García-Nieto, C-299/14, EU:C:2016:114, Rn. 47.

${ }^{86}$ EuGH, García-Nieto (Fn. 85), Rn. 48.

${ }^{87} \mathrm{EuGH}$, García-Nieto, (Fn. 85), Rn. 29.

${ }^{88}$ Siehe auch Farahat, (Fn. 75), $49 \mathrm{ff}$. 
Vorschriften der Richtlinie dem Grundsatz der Verhältnismäßigkeit entsprechen, garantiert dies noch nicht, dass durch die Anwendung der jeweiligen Vorschriften auch ein dem Grundsatz der Verhältnismäßigkeit entsprechendes Ergebnis erzielt wird. Gesetzliche Vorschriften als abstraktgenerelle Regelungen können meist nicht alle spezifischen Umstände des jeweiligen Einzelfalles berücksichtigen. Deshalb ist es unerlässlich, dass eine Verhältnismäßigkeitsprüfung durchgeführt wird, der eine Prüfung der Umstände des Einzelfalles vorausgeht und bei der die widerstreitenden Belange gegeneinander abgewogen werden. Schließlich wird weder bei der Entscheidung in der Rechtssache Dano oder Alimanovic noch Peña-García deutlich, wie die in der Entscheidung angewendeten Vorschriften eine Berücksichtigung des Privat- und Familienlebens sicherstellen.

In der Rechtsprechung des EuGH zum Zugang zu beitragsunabhängigen Sozialleistungen finden (zumindest vorerst) der Grundsatz der (finanziellen) Solidarität von Staatsangehörigen des Aufnahmemitgliedstaates mit denen anderer Mitgliedstaaten sowie eine Prüfung der Umstände des Einzelfalles und eine Verhältnismäßigkeitsprüfung zunehmend weniger Beachtung.

\section{Bürgerschaft einer neuen, sozialeren Union?}

Auch wenn Azoulai im Jahre 2014 betonte, dass wir Zeuge eines Wechsel von einer bloß ökonomischen Union hin zu einer neuen, breiteren sozialen Union werden, die ihre Grundlagen in einer Reihe gemeinsamer Werte findet, wobei dieser Wechsel vor allem durch die Rechtsprechung des Europäischen Gerichtshofes ermöglicht wurde ${ }^{89}$, so weist die Rechtsprechung des EuGH zur Unionsbürgerschaft insbesondere in den letzten Jahren in den hier untersuchten Bereichen eher restriktive Züge auf.

Weniger skeptisch scheinen $\breve{S} a d l$ und Madsen, welche die Auswirkungen der Finanzkrise auf die Rechtsprechung des EuGH zur Unionsbürgerschaft untersuchen, diese Entwicklung zu sehen. Sie kommen zu dem Schluss, dass die Auswirkungen der Finanzkrise auf das „Unionsbürgerrecht“ (EU citizenship law) sorgfältig auf die Randbereiche des Begriffes begrenzt

${ }^{89}$ Azoulai (Fn. 59), S. 4. 
sind, höchstwahrscheinlich mit dem Ziel, den Kernbereich dieses Gebiets des Europarechts zu sichern. ${ }^{90}$ Hinsichtlich der Rechte von mobilen Unionsbürger*innen mit einem greifbaren wirtschaftlichen und kulturellen Kapital, die möglicherweise zur Wirtschaft des Aufenthaltsstaates beitragen können, sei die Rechtsprechung, so Šadl und Madsen, hingegen konstant. ${ }^{91}$ Beim Ausweisungsrecht weisen sie darauf hin, dass der EuGH den Mitgliedstaaten einen größeren Spielraum einräume. ${ }^{92}$ Der EuGH habe die "legal margins" der Unionsbürgerschaft nach der Krise neu verhandelt. ${ }^{93}$ Weiterhin unterscheiden sie zwischen den Kernrechten der Unionsbürgerschaft (dem Freizügigkeitsrecht) auf der einen Seite und denen mit diesen Kernrechten verbundenen Rechten, wie z.B. dem Zugang zu bestimmten Sozialleistungen oder den abgeleiteten Rechten von drittstaatsangehörigen Familienangehörigen von Unionsbürger*innen auf der anderen Seite. ${ }^{94}$ Dementsprechend führen sie aus, dass der EuGH höchstwahrscheinlich nicht $\mathrm{zu}$ Gunsten von drittstaatsangehörigen Familienmitgliedern entscheiden werde ${ }^{95}$, eine Entwicklung, die auch in der Rechtssache Onuekwere deutlich wurde. Andererseits weist Coutts zutreffend darauf hin, dass das Urteil in der Rechtssache Onuekwere nicht auf drittstaatsangehörige Familienmitglieder beschränkt ist, sondern ebenso auf Unionsbürger*innen angewendet werden kann, wie das Urteil in der Rechtssache M.G. belegt, in dem die Grundsätze der Onuekwere Entscheidung unmittelbar auf einen Unionsbürger übertragen wurden. ${ }^{96}$

Weiterhin muss darauf hingewiesen werden, dass es nicht nur um die „legal margins“ oder die Frage nach dem Kernbereich oder Randbereich der Unionsbürgerschaft geht, sondern vielmehr um Unionsbürger*innen und ihre (drittstaatsangehörigen) Familienmitglieder, die sich in (wirtschaftlich oder sozial) prekären Situationen befinden. Auch die Unterteilung in Kernrechte der Unionsbürgerschaft auf der einen Seite und die von diesen Kernrechten abgeleitete Rechte auf der anderen Seite ist in

${ }^{90}$ ŠadllMadsen (Fn. 18), 60.

${ }^{91}$ Šadl/Madsen (Fn. 18), 44.

92 Šadl/Madsen (Fn. 18), 53.

${ }^{93}$ Šadl/Madsen (Fn. 18), 44.

94 Šadl/Madsen (Fn. 18), 40.

${ }_{95}$ Šadl/Madsen (Fn. 18), 44.

${ }^{96}$ Coutts (Fn. 21), 536. 
gewisser Weise geeignet, über die Fragmentierung des grundlegenden und einheitlichen Status der Unionsbürgerschaft hinweg zu täuschen.

Schließlich fragen $\breve{S} a d l$ und Madsen, wieviel von den Seitenrändern („margins“) der Unionsbürgerschaft noch entfernt werden könne, bevor man nicht mehr von der Unionsbürgerschaft als einem fundamentalen Status sprechen kann. ${ }^{97}$ Zutreffend stellt Thym fest, dass die Kernaussage der Rechtssache Dano zu sein scheint, dass der Unionsbürgerstatus nicht so fundamental ist wie er in vorherigen Urteilen oft zu sein schien. ${ }^{98}$ Nicht nur die „Ränder“ der Unionsbürgerschaft werden reduziert oder neu verhandelt, vielmehr wird, wie Farahat zutreffend ausführt, die ,solidarische und inklusive Dimension der Unionsbürgerschaft neu verhandelt ${ }^{\star 999}$. Dabei sind nicht nur die Seitenränder des Unionsbürgerstatus betroffen, sondern vielmehr die Unionsbürgerschaft als solche, die nicht mehr ein grundlegender, mitgegebener Status zu sein scheint, sondern vielmehr, wie Spaventa und Kramer betonen, ein Status, den sich der/die einzelne Unionsbürger*in erst verdienen müsse. ${ }^{100} \mathrm{Zu}$ einer ähnlichen Einschätzung gelangt Coutts, wenn er die Unionsbürgerschaft als „BewährungsBürgerschaft" ${ }^{101}$ bezeichnet, und ausführt, dass die Gewährung der mit dieser Bürgerschaft verbundenen Rechte nicht nur davon abhängig gemacht wird, dass der/die Inhaber*in bestimmte wirtschaftliche Bedingungen erfüllt, sondern dass er oder sie auch ein fortgesetztes beanstandungsfreies Verhalten an den Tag legen muss. ${ }^{102}$

\section{Schlussbemerkungen}

Die Unionsbürgerschaft stellt einen einheitlichen Status dar, der jedoch unterschiedliche Hürden oder Zugangsvoraussetzungen aufstellt, bevor man ihn als mobile(r) Unionsbürger*in in Anspruch nehmen kann. Mobile Unionsbürger*innen müssen ihre (wirtschaftliche) Integration im Sinne einer wirtschaftlichen Unabhängigkeit unter Beweis stellen (Art. 7 der

\footnotetext{
97 Šadl/Madsen (Fn. 18), 44.

${ }^{98}$ Thym, (Fn. 19), 253.

${ }^{99}$ Farahat, (Fn. 75), 45.

${ }^{100}$ Siehe D. Kramer, Verdiend verblijf: EU-burgers en de sociale bijstand, Tijdschrift voor Europees en economisch recht, SEW 2016/25, 60 ff.; Spaventa (Fn. 12).

${ }^{101}$ Coutts (Fn. 21) 531, ,probationary citizenship“.

102 Coutts (Fn. 21), 531.
} 
Unionsbürgerrichtlinie), um sich auf die in der Unionsbürgerrichtlinie und die im Vertrag verankerten Rechte berufen zu können.

Die wirtschaftliche Integration des Unionsbürgers/der Unionsbürgerin ist jedoch nicht ausreichend, vielmehr muss er oder sie auch (rechtlich) integriert sein, im Sinne einer Anpassung an die Normen des Aufnahmemitgliedstaates. Wird nur eines dieser Kriterien nicht erfüllt, so kann die Berufung auf die mit dem Unionsbürgerstatus verbundenen Rechte versagt werden.

Die in der Unionsbürgerrichtlinie verwendeten Begriffe der Integration, des sozialen Zusammenhalts und der Solidarität spielen eine wichtige Rolle bei der Umgestaltung der Unionsbürgerschaft durch die Rechtsprechung des EuGH.

Sowohl dem in der Unionsbürgerrichtlinie verwendeten Begriff des sozialen Zusammenhalts sowie dem Begriff der Integration von Unionsbürger*innen wird durch die Rechtsprechung des EuGH eine andere Ausrichtung gegeben. Während die Präambel der Unionsbürgerrichtlinie einen rechtsbasierten Ansatz verfolgt, indem sie die Integration von Unionsbürger*innen in die Gesellschaft des Aufnahmemitgliedstaates als Ziel betrachtet, welches es durch das Mittel, oder wie es im 18. Erwägungsgrund heißt, durch das „Instrument“ des Daueraufenthaltsrechts zu fördern gilt, wählen der EuGH und der Generalanwalt in jüngeren Entscheidungen und Schlussanträgen zum Daueraufenthaltsrecht beziehungsweise Ausweisungsrecht einen anderen Ansatz. Dieser Ansatz besteht darin, dass sie Integration - basierend auf der eingangs dargestellten zweiten Perspektive - dahingehend interpretieren, dass die Integration des Unionsbürgers/ der Unionsbürgerin zur Voraussetzung für die Inanspruchnahme einiger der mit der Unionsbürgerschaft verbundenen Rechte erklärt wird. Aus dem Ziel der Integration wird eine Voraussetzung, eine Hürde, die Unionsbürger*innen nehmen müssen. Nur wenn sich der/die Unionsbürger*in integriert und dadurch zum sozialen Zusammenhalt beiträgt, kann er oder sie die im AEUV und in der Unionsbürgerrichtlinie verankerten Rechte in Anspruch nehmen bzw. werden sie ihm oder ihr nicht nachträglich (im Falle der Ausweisung) entzogen. Unionsbürger*innen müssen somit in Vorleistung treten, um ihren Status vollumfänglich nutzen zu können. Durch diese Umkehrung werden nicht nur die Zielvorstellungen des sozialen Zusammenhalts und der Integration von Unionsbürger*innen, wie sie in der Unionsbürgerrichtlinie Eingang gefunden haben, verändert. Vielmehr ist diese konzeptionelle Kehrtwende in der Rechtsprechung des EuGH geeignet den sozialen Zusammenhalt selbst zu untergraben. 
Gemeinsame Werte, auf denen die Union gegründet ist ${ }^{103}$, wie der Grundsatz der Solidarität, dessen Stärkung den sozialen Zusammenhalt in der Europäischen Union fördern könnte, treten in der jüngeren Rechtsprechung des EuGH zur Unionsbürgerschaft in den Hintergrund. Der vom EuGH aufgestellte Grundsatz der (finanziellen) Solidarität der Staatsangehörigen des Aufnahmemitgliedstaates mit denen anderer Mitgliedstaaten, welcher bereits in der Grzelczyk-Entscheidung aus dem Jahr 2001 enthalten war und noch 2013 in der Brey-Entscheidung aufgegriffen wurde, verliert zunehmend an Bedeutung, indem er in aktuellen Entscheidungen 104 keinen Eingang $\mathrm{mehr}$ in die Entscheidungsgründe findet und damit offenbar keine Rolle mehr bei der Bestimmung des Inhalts der Unionsbürgerschaft spielt.

Die Freizügigkeit der Unionsbürger*innen könnte, wie Verschueren hervorhebt, zu einem Recht derjenigen werden, die das Glück haben, nicht auf Solidaritätsmechanismen angewiesen $\mathrm{zu}$ sein. ${ }^{105} \mathrm{Die}$ Unionsbürgerschaft scheint damit zu einem Status umgestaltet zu werden, der nur den wirtschaftlich aktiven, fleißigen oder wohlhabenden bzw. den gesellschaftlich integrierten Unionsbürger*innen ${ }^{106}$ zugutekommt und damit zu einer „Bewährungs-Bürgerschaft“ für alle diejenigen zu werden, die diese „Voraussetzungen“ (noch) nicht erfüllen. ${ }^{107}$

Diese Entwicklung begünstigt eine soziale Exklusion und eine fortschreitende Marginalisierung bestimmter Gruppen von Unionsbürger*innen ${ }^{108}$ und rückt damit die Frage nach den Zielen und dem Charakter der Europäischen Union in den Vordergrund, eine Frage, die sicherlich auch von anderen Institutionen aufgegriffen werden muss.

\footnotetext{
103 Art. 2 EUV.

${ }^{104}$ EuGH, Dano (Fn. 17), EuGH, Alimanovic (Fn. 84), EuGH, García-Nieto (Fn. 85); siehe auch EuGH, Kommission gegen Vereinigtes Königreich, C-308/14, EU:C:2016:436.

105 Verschueren (Fn. 80), 381.

${ }^{106}$ Spaventa (Fn. 12); siehe auch Thym (Fn. 17), 133, der ausführt, dass es sich um ein Freizügigkeitsregime handelt, „das die wirtschaftliche Aktivität prämiert und damit im Kern eine Freizügigkeit der mobilen und fleißigen Unionsbürger darstellt".

107 Coutts (Fn. 21), 531

${ }^{108}$ Siehe auch Farahat (Fn. 75), 51. Farahat weist in Bezug auf den Ausschluss arbeitssuchender Unionsbürger*innen von der sozialen Grundsicherung auf die „Gefahr dauerhafter sozialer Exklusion im Aufenthalts-Mitgliedstaat" hin.
} 